\title{
Contribution A L'etude De La Dynamique Sedimentaire De La Plage De Saket (Bejaia) Dans Le Nord-Est Algerien
}

\author{
Fahima Fellah, PhD \\ Département des Sciences Biologiques, Faculté SNV-STU, \\ Université de Bordj Bou Arreridj, Algérie \\ Redha Djenidi, Professeur \\ Département des Sciences Agronomiques, Faculté SNV-STU, \\ Université de Bordj Bou Arreridj, Algérie
}

Doi:10.19044/esj.2019.v15n33p285 URL:http://dx.doi.org/10.19044/esj.2019.v15n33p285

\section{Résume}

Le présent travail se focalise sur l'analyse de la dynamique sédimentaire de la plage de Saket durant une période de faible agitation. Cette étude présente une méthodologie basée sur deux approches complémentaires : une caractérisation granulométrique des sédiments et une simulation statistique par l'analyse des tendances de transport sédimentaire (GSTA). Les analyses granulométriques des sédiments de surface de la plage de Saket montrent la prédominance des sables fins qui représentent $60,60 \%$ des observations, suivie par des sables moyens avec 33,33\%. Les accumulations sableuses de Saket sont également caractérisées par un sédiment assez bien trié $(75,75 \%)$ à assez peu trié $(18,18 \%)$. La répartition spatiale des sables marque l'existence d'un granoclassement décroissant des sédiments de la côte vers le large. Le modèle GSTA appliqué dans cette étude, a abouti à des résultats cohérents avec l'organisation de ces structures sédimentaires. Le type FB- est le plus dominant dans notre zone d'étude.

Mots clés: Granulométrie, Analyse des Tendances de Transport Sédimentaire (GSTA), Plage de Saket, Bejaia 


\title{
Contribution to the Study of the Sedimentary Dynamics of the Saket Beach (Bejaia) in Northeastern of Algeria
}

\author{
Fahima Fellah, PhD \\ Département des Sciences Biologiques, Faculté SNV-STU, \\ Université de Bordj Bou Arreridj, Algérie \\ Redha Djenidi, Professeur \\ Département des Sciences Agronomiques, Faculté SNV-STU, \\ Université de Bordj Bou Arreridj, Algérie
}

\begin{abstract}
The present study focuses on the analysis of the sedimentary dynamics of the Saket beach during a period of low agitation. This study presents a methodology based on two complementary approaches: a granulometric characterization of the sediments and a statistical simulation by Grain size trend analysis model (GSTA). The results of the grain size analysis of the superficial sediments of the Saket beach demonstrated that there is predominance of fine sands by $60,60 \%$ of observation and the medium sands by $33,33 \%$. The sandy accumulaions has also been characterized by sediment moderately well sorted $(75,75 \%)$ to moderately sorted $(18,18 \%)$. The spatial distribution of the sands shows the existence of a fading classification of the sediments from the coast towards the wide one. Grain-size trend analysis of the sediment samples shows that there is coherence with the organization of sedimentary pattern. The FB- patterns is mostly represented in our study area.
\end{abstract}

Keywords: Granulometry, Grain Size Trend Analysis (GSTA), Saket beach, Bejaia

\section{Introduction}

Les plages, frontières entre la mer et la terre, représentent aujourd'hui à la fois une barrière contre les flots et un espace qui participe au développement local des communes, notamment par l'activité touristique balnéaire. Pourtant la plage en tant que stock de sable est dynamique, en équilibre précaire entre les "arrivées" et les "départs" de matériaux (PupierDauchez, 2008). Cette recherche d'équilibre dynamique se répercute sur la position du trait de côte dont le recul en direction des terres s'avère problématique lorsque ces espaces sont habités, aménagés par l'homme 
(Pupier-Dauchez, 2008). L'aménagement du littoral nécessite une bonne connaissance de sa reconfiguration qui constitue un préalable très important pour une meilleure gestion de cet environnement. La connaissance de la nature des sédiments superficiels est un élément indispensable à une bonne gestion du domaine côtier. En effet, la taille des grains est l'une des propriétés physiques la plus importante des sédiments et peut révéler des informations importantes sur la source, les antécédents de transport et la situation de la sédimentation (Folk \& Ward, 1957 ; Blott \& Pye, 2001). La caractérisation granulométrique des sédiments de surface a fait l'objet de nombreuses études (López, 2017 ; Venkatramanan et al., 2013 ; Amani et al., 2010 ; Touré et al., 2008 ; Akouango et al., 2000). Des études similaires ont été réalisées au Maghreb (Bellahbib et al., 2015 ; Brahim \& Chkioua, 2007 ; Mhammdi et al., 2005 ; Achab et al., 2005 ; Saidi et al., 2004). Cependant, Holland et Elmore (2008) ont montré que l'influence des propriétés hétérogènes des sédiments sur les processus côtiers était généralement sous-estimée en raison des difficultés rencontrées pour caractériser et quantifier les différents types de sédiments. L'application d'analyses statistiques étendues et multivariées de la distribution granulométrique est efficace pour identifier des similitudes et des différences discrètes entre des populations de sédiments mixtes (Nelson et al., 2014). Dans ce cadre, l'analyse des tendances de transport sédimentaire (GSTA) est une excellente approche pour établir le transport des sédiments dans divers environnements et a fait l'objet de nombreux travaux de recherches (Yamashita et al., 2018 ; Aouiche et al., 2016 ; O'Shea \& Murphy, 2016; Du et al., 2015 ; Sanchez \& Carriquiry, 2012 ; Jia et al., 2006 ; Héquette et al., 2005). Dans le présent travail, une étude sédimentaire de la plage de Saket (Bejaia) a été réalisée dans le but de déterminer d'une part les caractéristiques granulométriques et d'autre part, le sens et la direction du transit sédimentaire en utilisant le modèle GSTA.

\section{Matériel et méthodes : Echantillonnage}

Un total de 66 échantillons a été prélevé au niveau de la plage de Saket $\left(4^{\circ} 56 \mathrm{~W}\right.$ et $\left.4^{\circ} 57 \mathrm{E}\right)$ à environ $20 \mathrm{~km}$ à l'ouest de la ville de Bejaia dans le Nord-est de l'Algérie (Figure 1), durant deux sorties faites séparément les 18 et 20 novembre 2009, dans les mêmes conditions météorologiques de faible agitation (mer calme). D'un point de vue morphologique, la région concernée par l'étude s'inscrit dans une côte rocheuse abritant des plages à galets. 


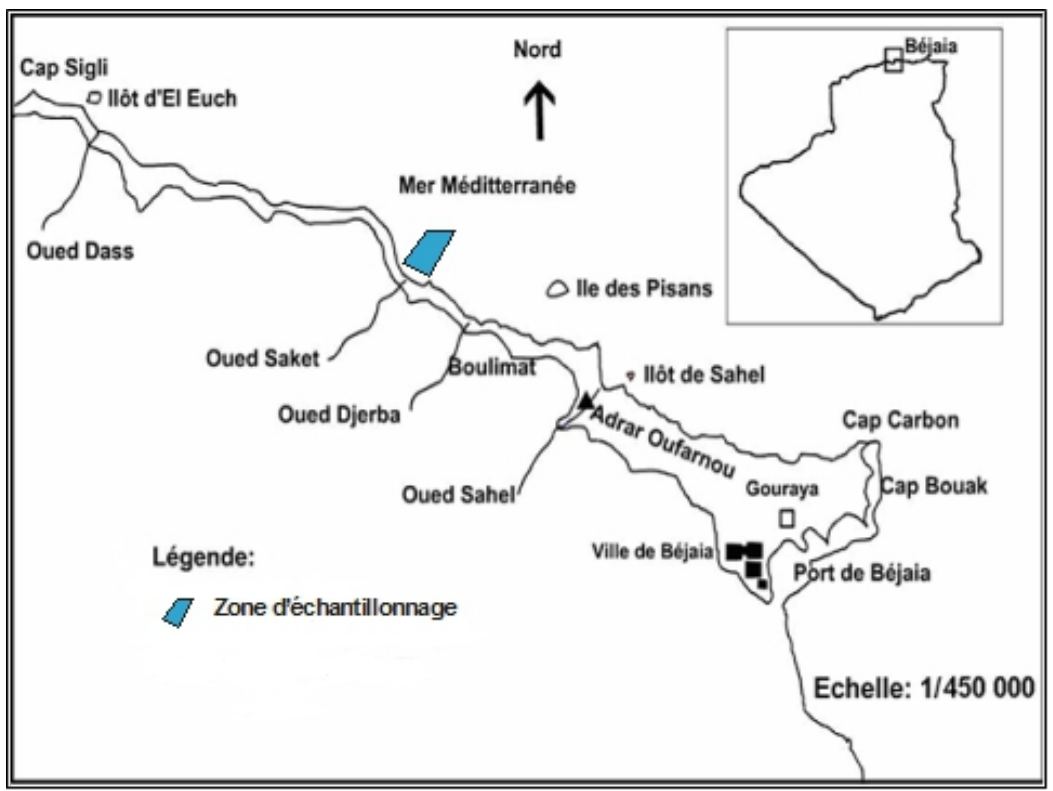

Figure 1 : Localisation géographique de la zone d'étude

Les sédiments de surface (environ $5 \mathrm{~cm}$ ) ont été récoltés sur le fond à l'aide d'une benne de type Van Venn à des profondeurs variant entre 3 et 28 $\mathrm{m}$. Chaque échantillon a été géoréférencé au moment du prélèvement à l'aide d'un GPS à main de type Explorist 100. Ensuite, ces données ont été injectées dans le logiciel Surfer 8.0 (Golden Software®) pour pouvoir schématiser leurs positionnements (Figure 2).

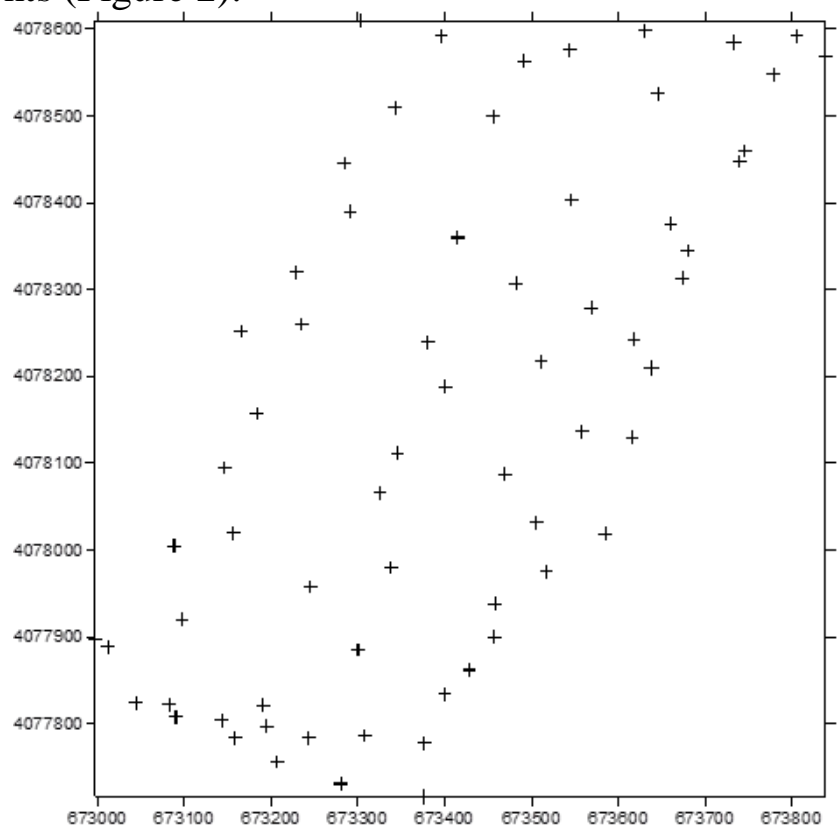

Figure 2. Localisation des prélèvements sédimentaires 


\section{Analyse granulométrique}

L'analyse granulométrique a été réalisée par un tamisage à sec sur une série de tamis complète, représentative de la classe des sables. La sélection des tamis a été faite selon la classification de Wentworth (1922). Les ouvertures des tamis vont de $0,063 \mathrm{~mm}$ à $2 \mathrm{~mm}$. Trois paramètres granulométriques ont été calculés à partir de la méthode graphique décrite par Folk \& Ward (1957), à savoir : la taille moyenne des sédiments $(\mu)$, l'indice de tri $(\sigma)$ et l'indice de symétrie de la distribution (Sk). L'unité Phi $(\Phi)$, qui correspond au système d'unité anglo-saxonne, a été choisie pour représenter les paramètres granulométriques. Cette unité est une transformation logarithmique de base 2 des diamètres de particules exprimées en millimètres selon la formule : Phi = $-\log 2(\mathrm{D})$.

\section{Méthode de calcul des tendances de transport sédimentaire (GSTA)}

Dans cette étude, la méthode de Gao et Collins (1992) a été adoptée pour analyser les voies de transport sédimentaire. Les principes théoriques de cette méthode peuvent être résumés comme suit : les paramètres d'un échantillon sont comparés avec ceux de chaque point environnant, 2 à 2, dans un rayon inférieur ou égal à une distance critique (DC) qui correspond à la distance maximum séparant deux points adjacents. $\mathrm{Si}$ un point d'échantillonnage génère des vecteurs dans plusieurs directions, une sommation finale est faite de manière à obtenir un vecteur unique $\vec{R}(\mathrm{x}, \mathrm{y})$ résultant tel que:

$$
\vec{R}(x, y)=\sum_{1}^{n} \vec{r}(x, y)_{i}
$$

Une opération de filtrage est appliquée pour réduire une nouvelle fois le bruît causé par les vecteurs dont les directions sont éloignées de la direction générale du modèle. Les vecteurs de transport sont obtenus sur la base de l'équation suivante :

$$
\vec{R}_{\text {filt }}(x, y)=1 /(K+1) \times\left[\vec{R}(x, y)+\sum_{1}^{K} \vec{R}_{J}\right]
$$

Le programme GiSedTrend (Gis based sediment trend analysis), développé en langage C++ par Poizot et Méar (2010), a été utilisé pour calculer les tendances du transport sédimentaire

$$
\vec{R}(x, y)=\sum_{1}^{n} \vec{r}(x, y)_{i}
$$




\section{Résultats et discussion :}

La plage est composée dans sa globalité par une majorité de sables fins qui représentent $60,60 \%$ des observations. Les sables moyens constituent $33,33 \%$ de la totalité des échantillons récoltés et les sables grossiers 4,55\% (Figure 3). Les accumulations sableuses de la plage de Saket sont également caractérisées par un sédiment assez bien trié (75,75\% des observations) à assez peu trié $(18,18 \%)$ (Figure 3). Les distributions presque symétriques représentent 46,96 \% des observations, alors que les distributions asymétriques prononcées vers les particules grossières en représentent 36,36 $\%$ (Figure 3).

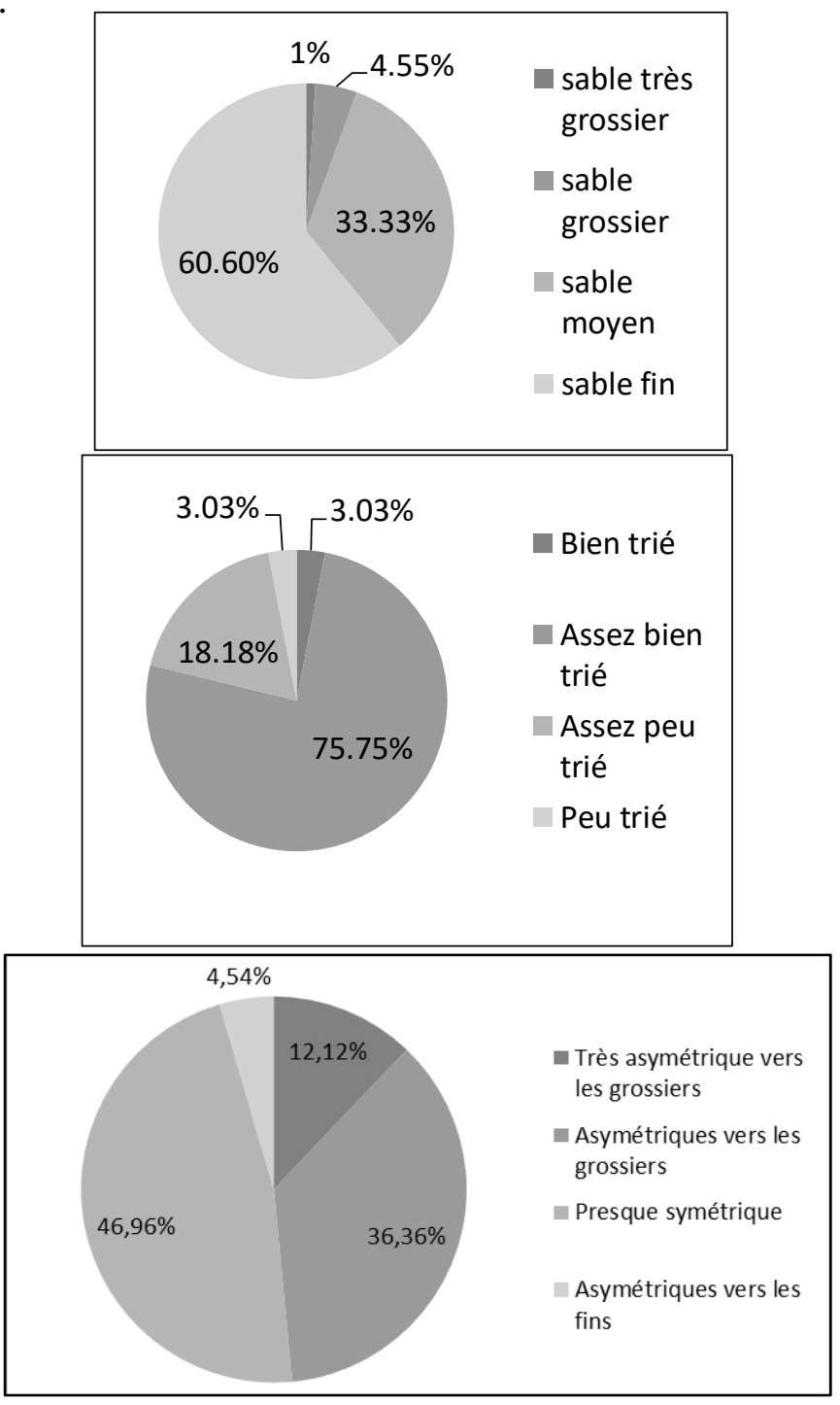

Figure 3. Répartition des paramètres granulométriques sur l'ensemble des 66 échantillons récoltés 
Les mesures de bathymétrie réalisées pendant la campagne de prélèvement des sédiments sont présentées dans la Figure 4 (isobathes). La carte bathymétrique montre des isobathes parallèles au trait de côte avec des petites inclinations dans la partie Sud-est. En allant de la côte vers le large, le fond marin est un peu profond surtout dans sa partie sud-est (de 0 à $-8 \mathrm{~m})$. Vers le large, le fond marin descend de façon régulière et assez rapidement à des profondeurs allant jusqu'à $28 \mathrm{~m}$.

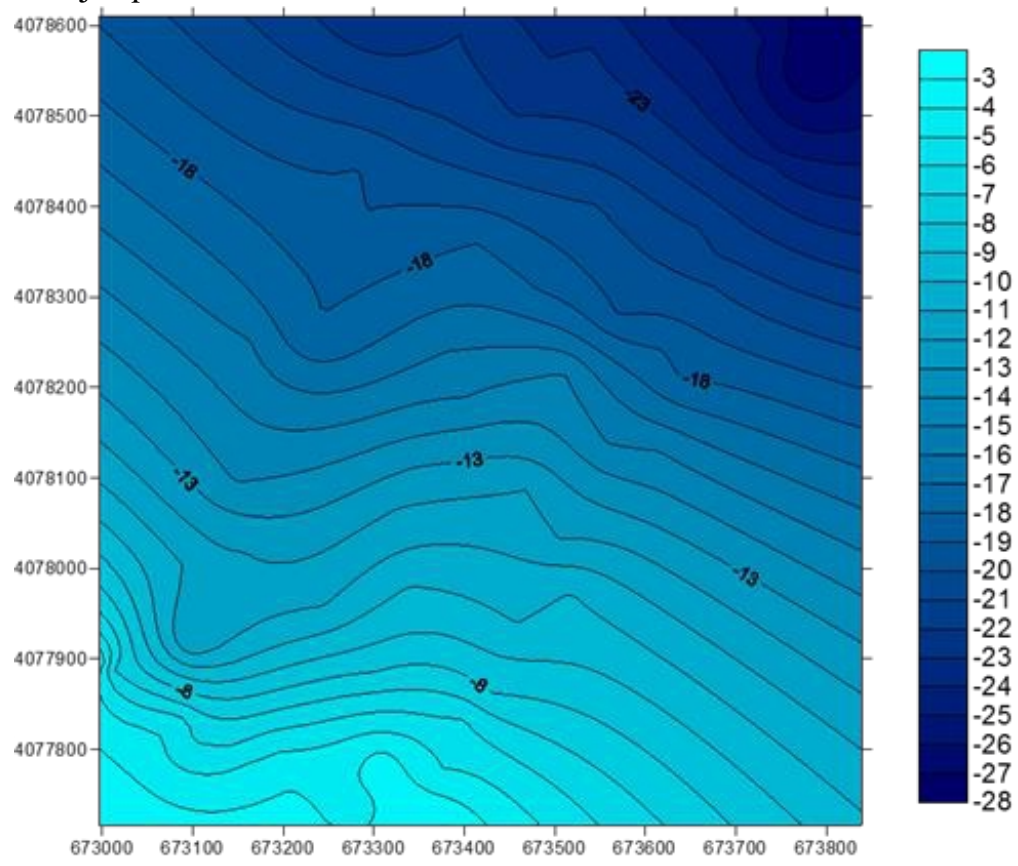

Figure 4. Evolution de la bathymétrie au niveau de la plage de Saket

Les sédiments grossiers sont localisés en bord de plage et beaucoup plus dans la partie Sud-ouest à une profondeur comprise entre 0 et $8 \mathrm{~m}$ (Figure 5). Les sables moyens sont, pour l'essentiel, localisés entre les isobathes -8 et $-13 \mathrm{~m}$. Cependant, on remarque la présence de cette fraction moyenne à une profondeur dépassant les $25 \mathrm{~m}$ dans la partie Nord-est. Les sables fins deviennent dominants au-delà de l'isobathe $-13 \mathrm{~m}$. 


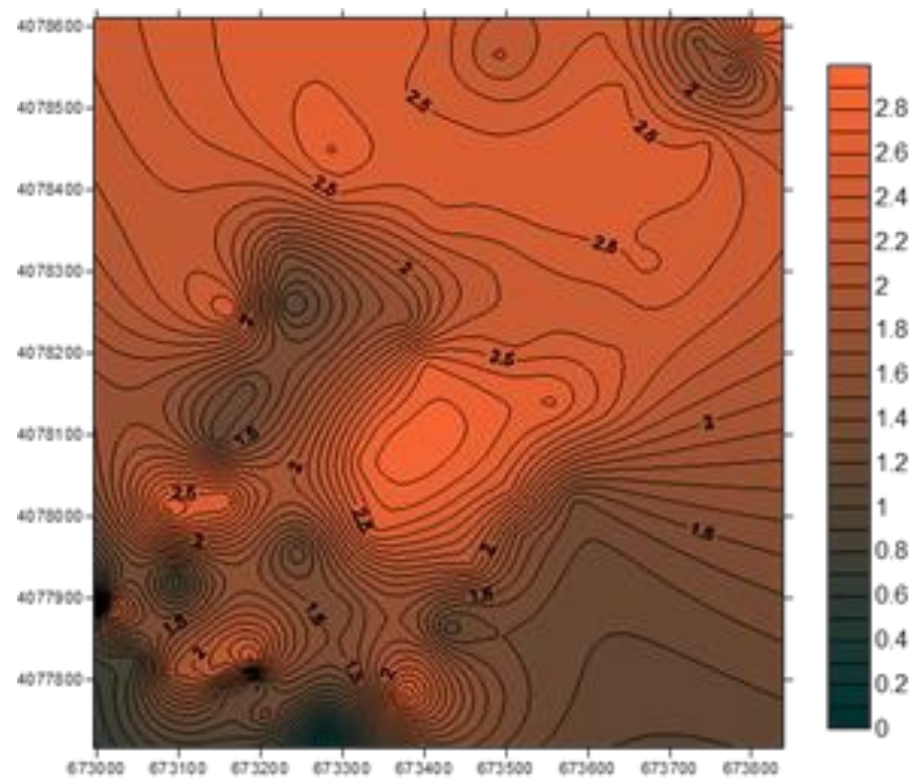

Figure 5. Distribution de la taille moyenne des grains au niveau de la plage de Saket

Les sédiments assez bien triés se trouvent sur la plus grande partie de la surface échantillonnée, alors que les sédiments assez peu triés se trouvent dans la partie Ouest de cette dernière (Figure 6). Le tri sédimentaire apparaît d'autant plus important que les particules sont fines, à l'exception de la limite Sud-ouest de notre site qui présente un tri qui s'améliore vis à vis des particules grossières. La faible fraction de sable qui présente un caractère mal trié se trouve dans la limite Nord-est de la zone d'étude.

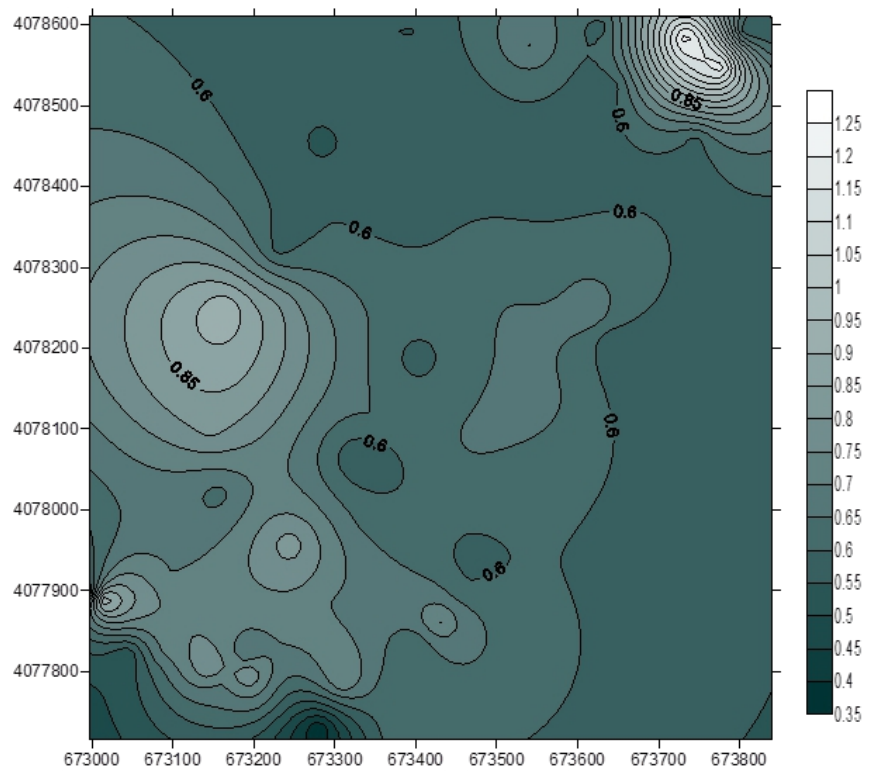

Figure 6. Evolution du coefficient de classement- Sorting- au niveau de la plage de Saket 
La figure 7 montre que le Skewness diminue en allant de la limite Sudouest vers la limite Nord-est, ce qui veut dire que les sables deviennent de plus en plus asymétriques vers les grossiers. L'asymétrie des accumulations sableuses est d'autant plus prononcée vers les particules grossières que la taille moyenne du sédiment diminue.

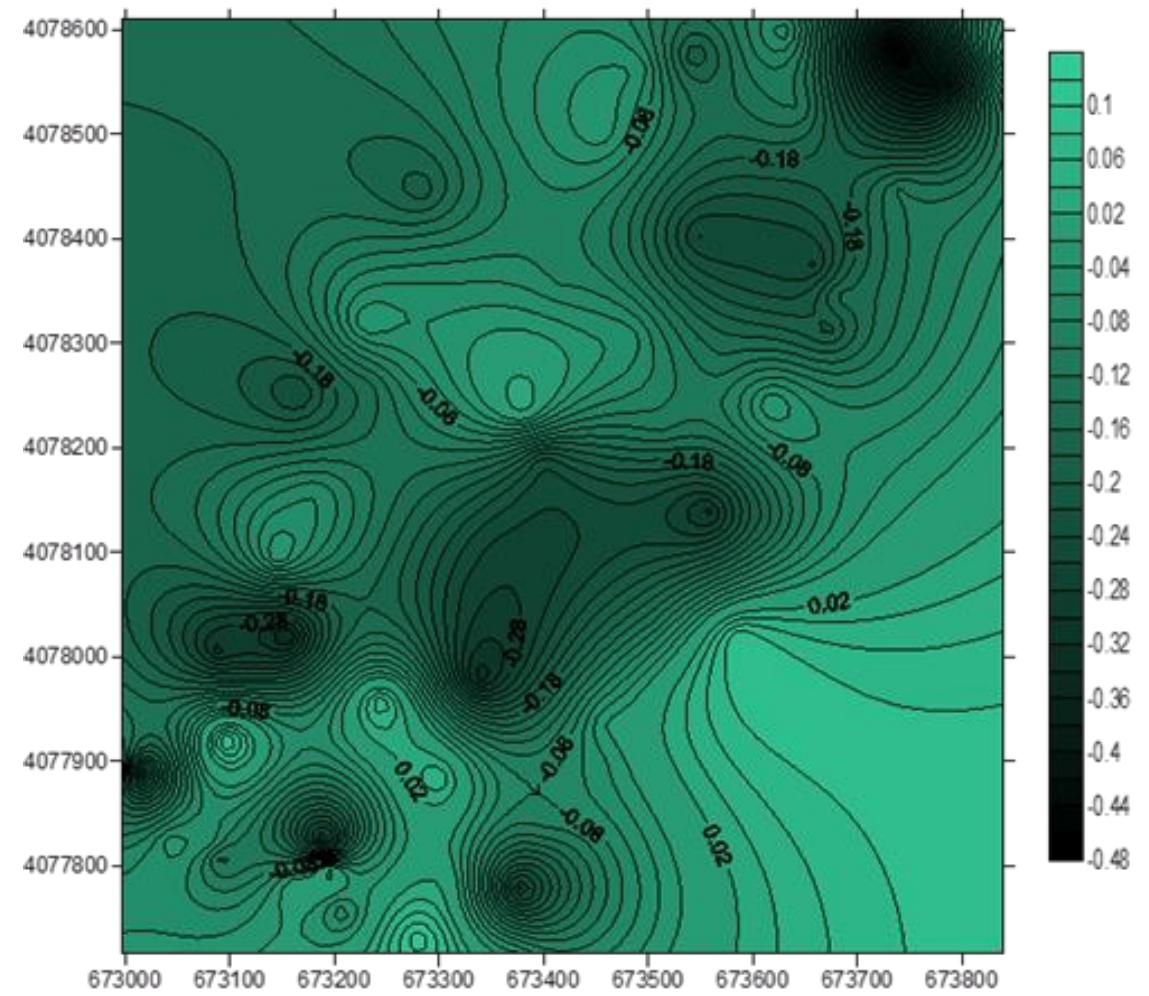

Figure 7. Evolution du coefficient d'assymétrie - Skewness- au niveau de la plage de Saket

Les tendances du transport sédimentaire sont calculées à partir des cartes de distribution des trois paramètres granulométriques, en prenant une distance critique de 120 mètres. Les deux cas de transport enregistrés dans notre zone sont FB- et $\mathrm{CB}+$. La majorité des tendances sur les plages ont coïncidé avec ces deux cas originaux spécifiés par McLaren (1981). Dans le cas FB-, les sédiments sont érodés de manière sélective et ces changements de texture, reflétés dans le matériau transporté, peuvent être préservés avec un dépôt presque complet alors que dans le cas $\mathrm{CB}+$, les sédiments transportés subissent un dépôt sélectif des fractions les plus grossières en raison de la modification de l'énergie des processus de transport (Kairyte \& Stevens, 2015). Deux directions de transport se distinguent dans cette analyse (Figure 8). Un transport offshore, en direction du large et essentiellement perpendiculaire à la plage avec deux directions privilégiées : $\mathrm{N}$ et $\mathrm{NE}$ et un transport onshore, en direction de la côte, dans un sens inverse au premier, c'est-à-dire qu'il va dans la direction S et SW. 


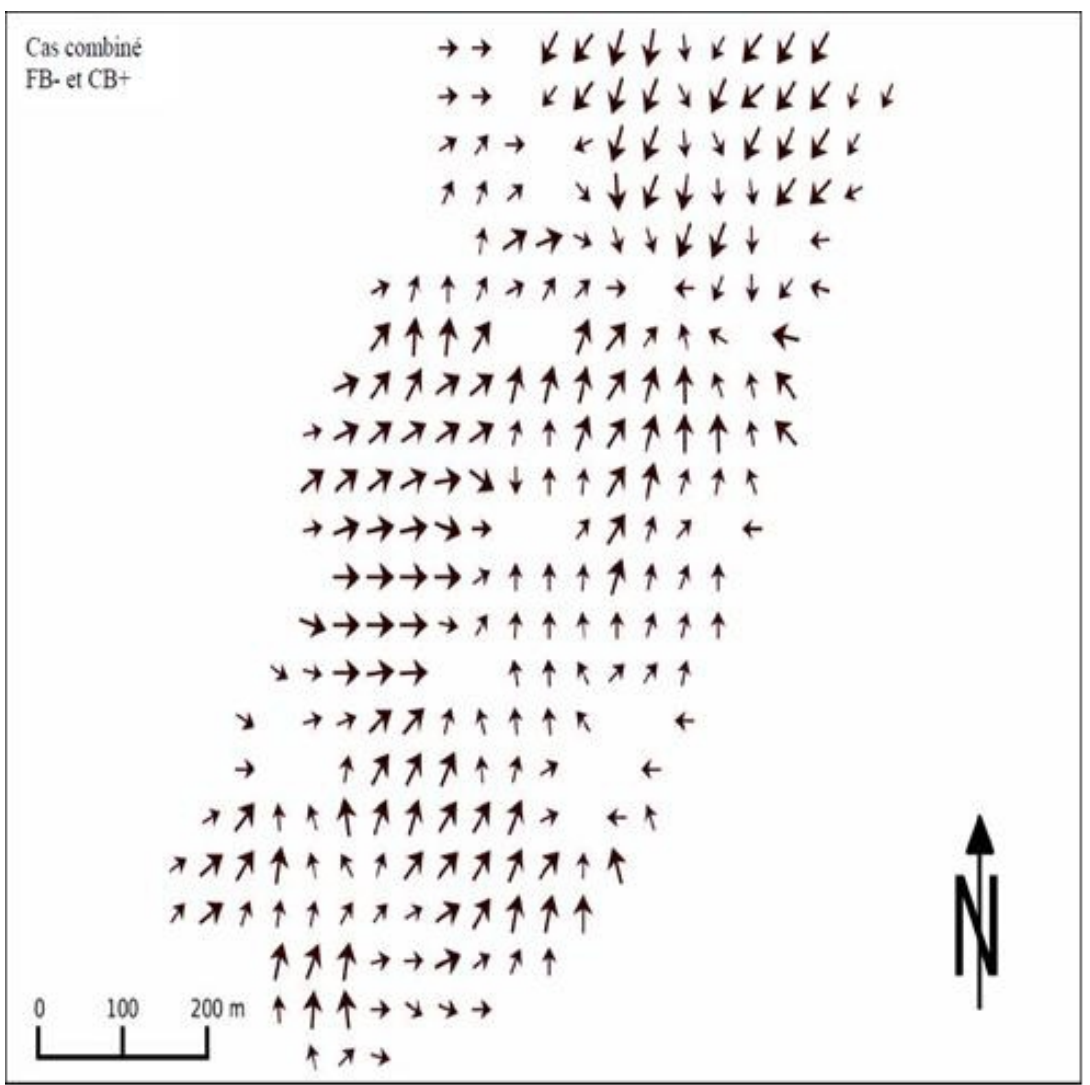

Figure 8. Tendances de transport sédimentaire dans la plage de Saket.

En observant le sens des transits sédimentaires, le cas FB- est indiqué comme le plus dominant. Le cas de dépôt FB- est connu dans les environnements à basse énergie, où il y a peu de chance pour un dépôt sélectif et les sédiments sont laissés derrière pendant un seul événement sédimentaire (Kairyte \& Stevens, 2015). Selon Storlazzi et al. (2004), ce type est à l'origine d'un courant de compensation qui prend place sur le fond et agit en direction opposée du flux initial, c'est à dire vers le large. Cependant, pour notre cas, l'absence d'une agitation significative liée au vent exclurait l'implication de celui-ci dans les transports potentiels prédits. Il s'agirait vraisemblablement d'un courant de densité assez fort pour pouvoir exercer un cisaillement au niveau du fond sédimentaire dépassant le seuil nécessaire pour le début de l'entrainement des sédiments. D'après Cordier (2007), les processus de transport de faible énergie, caractérisés par les tendances de type FB-, traduisent une érosion des particules sédimentaires à leur source, puis un transport suivi d'un dépôt total. Ce processus pourrait expliquer le phénomène de l'érosion marine que connait la région de Saket depuis quelques années. La présence du type $\mathrm{CB}+$ qui est dû à des processus de forte énergie, indique probablement que les conditions de mauvais temps qui ont régné dans notre 
région d'étude quelques jours avant l'échantillonnage, ont eu une influence sur le sens du transport sédimentaire.

\section{Conclusion}

La dynamique sédimentaire relative de la plage de Saket obtenue par la méthode GSTA a permis d'observer un transport sédimentaire potentiel essentiellement dirigé vers le $\mathrm{NE}$ avec quelques transports dirigés vers le large. Ces derniers seraient induits par des courants de densité assez forte pour pouvoir exercer un cisaillement au niveau du fond sédimentaire dépassant le seuil nécessaire pour le début de l'entrainement des sédiments. Enfin, ces résultats qualitatifs ne sont qu'une première approche. Ils doivent être complétés par des mesures hydrodynamiques afin de mettre en évidence avec des observations réelles les différents courants qui agissent et interagissent au niveau de l'avant-côte de la zone d'étude et ce, en vue de proposer un plan d'aménagement côtier permettant la conservation et la protection du littoral de Bejaia.

\section{References:}

1. Achab, M., El Moumni, B., El Arrim, A., \& Gutierrez Mas, J.M. (2005). Répartition des faciès sédimentaires récents en milieu marin côtier : exemple des baies de Tanger (NW-Maroc) et de Cadix (SWEspagne). Bulletin de l'Institut Scientifique, 27: 1-9.

2. Akouango, E., Certain, R., Barusseau, J.P. (2000). Mobilité sédimentaire: définition par simulation des populations granulométriques mobiles sur l'avant-côte. VIème Journées Nationales Génie Civil- Génie Côtier, Caen, 185-196.

3. Amani, E.M.E., Monde, S., Nguessan, Y.A., \& Aka, K. (2010). Analyse sédimentologique et diffractométrique des sédiments superficiels de la lagune Digboué de San Pedro (Côte d'Ivoire). European Journal of Scientific Research, 44(4): 527-540.

4. Aouiche, I., Daoudi, L., Ihbach, F.Z., \& Ouahmane, B. (2016). Caractérisation minéralogique et granulométrique des sédiments littoraux de la baie d'Agadir (Maroc atlantique): dynamique sédimentaire et sources des matériaux. Journal of Materials and Environmental Science, 7 (10): 3523-3535.

5. Bellahbib, N., Rezqi, H., Oujidi, M., \& Bengamra, S. (2015). Etude granulométrique et minéralogique des sédiments superficiels du littoral de Saïdia et de l'estuaire de la Moulouya (Nord-Est du Maroc). LARHYSS Journal, 12(4): 19-40.

6. Blott, S.J., \& Pye, K. (2001). GRADISTAT: A grain size distribution and statistics package for the analysis of unconsolidated sediments. Earth Surface Processes and Landforms, 26 (11): 1237-1248. 
7. Brahim, M., \& Chkioua, A. (2007). Répartitions granulométriques et minéralogiques des sédiments de surface dans le golfe de Tunis. Bulletin de l'Institut National des Sciences et Technologies de la Mer (INSTM), Vol. 34.

8. Cordier, E. (2007). Dynamique hydrosédimentaire du récif frangeant de l'Hermitage / La Saline (La Réunion) : Processus physiques et flux sédimentaires. Thèse de doctorat, Université de la Réunion, 193 pp.

9. Du, X., Gama, C., Liu, J.T., \& Baptista, P. (2015). Sediment sources and transport pathway identification based on grain-size distributions on the SW coast of Portugal. Terrestrial, Atmospheric and Oceanic Sciences, 26 (4): 397-409.

10. Folk, R.L., \& Ward, W.C. (1957). Brazos river bar: a study in the significance of grain size parameters. Journal of Sedimentary Petrology, 27(1): 3-26.

11. Gao, S., \& Collins, M. (1992). Net sediments transport patterns inferred from grain-size trends, based upon definition of "tranport vectors". Sedimentary Geology, 80: 47-60.

12. Héquette, A., Hemdane, Y., \& Anthony, E.J. (2008). Determination of sediment transport paths in macrotidal Shoreface environments: a comparison of grain-size trend analysis with near-bed current measurements. Journal of Coastal Research, 24(3): 695-707.

13. Holland, K.T., \& Elmore, P.A. (2008). A review of heterogenous sediments in coastal environments. Earth Science Review, 89(3): 116134.

14. Jia, J., Wang, Y., Gao, S., Wang, A., \& Li, Z. (2006). Interpreting grain-size trends associated with bedload transport on the intertidal flats at Dafeng, central Jiangsu coast. Chinese Science Bulletin, 51(3): 341-351.

15. Kairyte, M., \& Stevens, R.L. (2015). Composite methodology for interpreting sediment transport pathways from spatial trends in grain size: A case study of the Lithuanian coast. Sedimentology, 62(3): 681696.

16. López, G.I. (2017). Grain size analysis. Encyclopedia of Geoarchaeology, Allan S. Gilbert (Volume Ed.), 341-348.

17. McLaren, P. (1981). An interpretation of trends in grain size measures. Journal of Sedimentary Research, 51: 611-624.

18. Mhammdi, N., Achab, M., Hamoumi, N., \& Azza, A. (2005). Les sables titanifères du littoral d'Azemmour et de l'estuaire de l'Oum ErRbia (côte atlantique marocaine): sédimentologie et potentiel d'exploitation. Bulletin de l'Institut Scientifique, 27: 83-91. 
19. Nelson, P.A., Bellugi, D., \& Dietrich, W.E. (2014). Delineation of river bedsurface patches by clustering high-resolution spatial grain size data. Geomorphology, 205: 102-119.

20. O'Shea, M., \& Murphy, J. (2016). The validation of a new GSTA case in a dynamic coastal environment using morphodynamic modelling and bathymetric monitoring. Journal of marine Science and Engineering, 4(1), 1-15.

21. Poizot, E., \& Mear, Y. (2010). Using a GIS to enhance grain size trend analysis. Environmental Modelling \& Software, 25(4): 513-525.

22. Pupier-Dauchez, S. (2008). Le rechargement sédimentaire des plages vendéennes et charentaises : vers une gestion globale du littoral. Actes du colloque international pluridisciplinaire "Le littoral : subir, dire, agir", Lille, 11 pp.

23. Saidi, H., Brahim, M., \& Gueddari, M. (2004). Caractérisation granulométrique et minéralogique des sédiments de surface de la frange littorale Sidi Bou Saïd - La Goulette. Bulletin de l'Institut National des Sciences et Technologies de Mer de Salammbô, 31: 97106.

24. Sánchez, A., \& Carriquiry, J. (2012). Sediment transport patterns in Todos Santos Bay, Baja California, Mexico, inferred from grain-size trends. In: Mannig A. (ed) Sediment transport in Aquatic environments. Croatia: IntechOpen, pp. 1-18.

25. Storlazzi, C.D., Ogston, A.S., Bothner, M.H., Field, M.E., \& Presto, M.K. (2004). Wave and tidally driven flow and sediment fluxes across a fringing coral reef: Southern Molokai, Hawaii. Continental Shelf Research, 24(12):1397-1419.

26. Touré, M., Affian, K., Djagoua, E.M’V., Aka, K., \& Diaby, S. (2008). Influence de la morphologie et de la sédimentologie dans le choix d'une technique de protection des côtes : cas du segment de côte d'Assouinde (Assinie, Côte d'ivoire). European Journal of Scientific Research, 21: 650-661.

27. Venkatramanan, S., Ramkumar, T., \& Anithamary, I. (2013). Distribution of grain size, clay mineralogy and organic matter of surface sediments from Tirumalairajanar Estuary, Tamilnadu, east coast of India. Arabian Journal of Geosciences, 6 (5): 1371-1380.

28. Wentworth, C.K. (1922). A scale of grade and class terms for clastic sediments. Journal of Geology, 30(5): 377-392.

29. Yamashita, S., Naruse, H., \& Nakajo, T. (2018). Reconstruction of sediment-transport pathways on a modern microtidal coast by a new grain-size trend analysis method. Progress in Earth and Planetary Science, 5:7. 